Anuario Latinoamericano Ciencias Políticas

y Relaciones Internacionales

vol. 5, 2017

pp. 291-315

\section{Hacia una medición del poder naval en América Latina*}

\section{Towards the measurement of naval power in Latin America}

\author{
Noé Cuervo Vázquez ${ }^{* *}$ \\ CENTRO DE ESTUDIOS SUPERIORES NAVALES \\ SECRETARÍA DE MARINA - ARMADA DE MÉXICO, MÉXICO \\ Marcos Pablo Moloeznik $* * *$ \\ UNIVERSIDAD DE GUADALAJARA, MÉXICO \\ $\triangle$ mmoloeznik@yahoo.es
}

\section{RESUMEN}

A lo largo de esta contribución se lleva a cabo un esfuerzo por medir el poder naval de los países latinoamericanos con el objetivo de clasificar a las armadas de la región. Para ello, se parte de un proceso de selección de los indicadores más adecuados para cada dimensión de las variables independientes identificadas. Una vez seleccionados los indicadores, resulta necesario fijar los valores de ponderación de cada factor. Aquí, se debe establecer la medida estándar óptima de cada indicador evaluado, para que las medidas de los distintos países se comparen con el estándar óptimo y así obtener el valor del índice del poder naval; valor obtenido que se correlaciona con los indices del poder naval total de los otros países evaluados. El modelo generado para la simulación de los resultados es de ecuaciones lineales, considerando que no se contempla una retroalimentación dinámica de los datos.

PALABRAS CLAVE: poder naval, poder naval total, América Latina, capacidades navales actuales, capacidades navales potenciales.

\section{ABSTRACT}

This article attempts to measure the naval power of the Latin American countries in order to classify the navies of the region. To fulfill this objective, a selection process of the most appropriate indicators for each dimension of the independent variables identified was performed. Once the indicators have been selected, it was necessary

* El presente trabajo de investigación se basa en información consultada en fuentes abiertas especializadas en el análisis del poder naval mundial, y en momento alguno trata asuntos que tengan relación con las actividades clasificadas de la Secretaría de Marina - Armada de México (SEMAR), ni proporciona algún tipo de información clasificada de la propia SEMAR.

** Candidato a doctor en Educación por la Universidad Autónoma de Tamaulipas.

*** Profesor e investigador titular de la Universidad de Guadalajara, México. 
to set the weight of each factor. Then, the optimum standard measure of each evaluated indicator was established, so that the measures from different countries could be compared with this optimum standard and the index value of naval power could be obtained. In other words, the value that correlates the indices of all naval power of each evaluated country. The model generated for the simulations is a linear regression, and does not consider dynamic feedback data.

KEYWORDS: naval power, total naval power, Latin America, current naval capabilities, potential naval capabilities.

"A diferencia del ejército y de la fuerza aérea, cuyo tamaño y poder de fuego deben guardar relación con aquellos del adversario potencial, el tamaño de la armada es determiando por el quantum de activos e intereses marítimos que uno debe proteger."

Capitán de Fragata de Bangladesh Mohd Khursched Alam

\section{A manera de introducción}

El poder naval, armada o marina de guerra es un instrumento del poder de un Estado-nación, cuya finalidad es la protección y preservación de los activos marítimos, y sus actividades deben ser vistas de acuerdo con los recursos que se le destinan y las misiones que se le encomiendan (Sheina 1991: 15).

Cabe señalar que una marina de guerra, como se conoce tradicionalmente a la armada, es en primer lugar un conjunto de medios: buques, aeronaves, apoyo; y el personal necesario para servirlos. La primacía se suele dar en los medios sobre el personal; lo que no niega que este último constituya la mayor riqueza del poder naval, pero son los medios los que, a diferencia de las otras fuerzas armadas, determinan la estructura de la armada, su modelo (de Saint Salvy 1994: 57).

De donde se desprende la doctrina naval británica clásica, que clasifica a las armadas por el color de las aguas (Moloeznik 2013):

- Blue Water Navy: 1) Estados Unidos de Norteamérica; 2) Reino Unido de la Gran Bretaña, Francia, Rusia, India y Japón.

- Green Water Navy: 1) China y Brasil (potencias en ascenso) 2) Colombia, Venezuela y México (guardacostas con capacidad de proyección oceánica).

- Brown Water Navy: 1) Guatemala, Honduras y el Salvador (Fuerzas Navales) 2) Bolivia y Paraguay (Fuerzas Fluviales)

En ese marco, los portaaviones, los destructores y las fragatas son reconocidos como las principales fuerzas de combate de superficie de las armadas. ${ }^{1}$ Los buques constituyen la esencia misma de la armada. Una marina de guerra

1 Véase: "Navy" en: International Institute for Strategic Studies (IISS), The Military Balance 2013, Publisher Routledge, London 2013. 
podrá carecer de aeronaves, helicópteros, submarinos e infantería de marina, pero no puede concebirse una armada sin buques.

A partir de estas ideas-fuerza, la presente contribución tiene por objeto poner a consideración de lector un modelo de medición del poder naval, mediante la determinación de las capacidades reales y potenciales de las armadas de América Latina. En cuanto al empleo del poder naval, conviene abrevar en la propuesta teórica de Milan Vego (2003), para quien el empleo moderno de las fuerzas de combate -consecuencia de la evolución del arte de la guerra- se puede dividir en dos grandes grupos: acciones tácticas navales y grandes operaciones navales, las cuales tienen los siguientes propósitos y acciones:

- Propósitos: destruir la flota enemiga en la mar o sus bases, desembarcar anfibios en costas con oposición, destruir las instalaciones costeras enemigas, interrumpir el comercio marítimo enemigo, proteger el comercio marítimo propio, bloquear o contrabloquear, destruir fuerzas nucleares estratégicas embarcadas del enemigo, proteger las fuerzas nucleares embarcadas propias, apoyar a las fuerzas terrestres en la costa (Vego 2003: 184).

- Acciones: prevención del conflicto, protección del tráfico, libertad de navegación y sobrevuelo, operaciones de paz, conflictos de baja intensidad, asistencia humanitaria, auxilio en catástrofes naturales, búsqueda y salvamento (Vego 2003: 186).

Estos grupos de acciones permiten determinar, de manera objetiva, el nivel de dominio del mar y, con ello, generar un índice del poder naval de un Estado-nación a través de un modelo matemático.

Para dar cumplimiento al objetivo de esta contribución, se determinan dos grandes campos que permiten establecer el Poder Naval Total de un Estado, bajo un esquema estratégico-operacional: las Capacidades Navales actuales ${ }^{2}$ y las Capacidades Navales potenciales. ${ }^{3}$

En tanto que, para estar en condiciones para realizar el presente trabajo de investigación, se establecieron los siguientes parámetros:

- Como objeto de estudio se identifica al Poder Naval.

- Como variables se identifica sólo una variable dependiente y dos variables independientes:

Variable Dependiente: $\quad \mathrm{Vd} 1=$ Poder Naval total $(\mathrm{PNt})$.

Variables Independientes: $\quad$ Vil $=$ Capacidad Naval actual $(\mathrm{CNa})$

$\mathrm{Vi} 2=$ Capacidad Naval potencial $(\mathrm{CNp})$
Hacia una medición del poder naval en América Latina

Noé Cuervo Vázquez Marcos Pablo Moloeznik

\footnotetext{
2 Se refiere al valor de la capacidad operativa de las unidades de superficie, aéreas y submarinas adscritas a la marina de guerra, que determinan el dominio positivo o negativo del mar y está determinada por once dimensiones que se explican más adelante.

3 Es el valor de la capacidad de transformación de las capacidades operativas de la marina de guerra, a partir de factores no operativos del Estado, que tienen influencia en el aspecto naval y está determinada por diez dimensiones que son desarrolladas a continuación.
} 
La definición conceptual de las variables es como sigue:

Vd1 = Poder Naval total (Pnt) y es el valor de la capacidad bélica del Estado en la mar, expresada principalmente por los recursos de la marina de guerra. ${ }^{4}$

Vil = Capacidad Naval actual $(\mathrm{CNa})$ y es el valor de la capacidad operativa de las unidades de superficie, aéreas y submarinas adscritas a la marina de guerra, que determinan el dominio positivo o negativo del mar y está determinada por once dimensiones (Diez 2006) con sus indicadores que son mostrados en el siguiente acápite.

Vi2 = Capacidad Naval potencial $(\mathrm{CNp})$ y es el valor de la capacidad de transformación de las capacidades operativas de la marina de guerra, a partir de factores no operativos del Estado que tienen influencia en el aspecto naval (Moloeznik 2009) y está determinada por diez indicadores, en donde se describe el modelo de medición propuesto.

Mientras que la definición operacional de las tres variables es:

$\mathrm{Vd}=$ Poder Naval total $(\mathrm{PNt})^{5}$ y es el resultado del algoritmo que relaciona a la Capacidad Naval actual (CNa) con la capacidad Naval potencial (CNp), que se construyó durante el desarrollo de la presente investigación, y que se resolvió a partir de un instrumento informatizado desarrollado en el programa EXCEL 2013, quedando la fórmula sintetizada de la siguiente manera: $\mathrm{PNt}=\mathrm{CNa}+\mathrm{CNp} 2$

Vil = Capacidad Naval actual $(\mathrm{CNa})$ y se midió a partir de un algoritmo que relacionó a las once (11) dimensiones con sus indicadores que conforman el can; este algoritmo consiste en la suma algebraica de cada dimensión multiplicada por su ponderación horizontal y vertical, las cuales serán explicadas durante el desarrollo del presente trabajo de investigación.

$\mathrm{Vi} 2$ = Capacidad Naval potencial $(\mathrm{CNp})$ y se midió a partir del algoritmo que relaciona a las diez (10) dimensiones con los indicadores que conforman el Cnp; este algoritmo consistirá en la suma algebraica de cada dimensión multiplicada por su ponderación horizontal únicamente, las cuales serán explicadas durante el desarrollo del presente trabajo de investigación.

\section{Modelo de medición del poder naval}

En este marco y a partir de un enfoque científico-cuantitativo, en primer lugar se lleva a cabo un proceso de selección de los indicadores más adecuados para cada dimensión de las variables independientes identificadas. Cabe

4 Esta definición está contenida en el "Glosario de términos unificados por personal de la Secretaría de la Defensa Nacional -SEDENA- y de la Secretaría de Marina (SEMAR)” del 14 de agosto del 2013, autorizado como doctrina por el Alto mando de la Secretaría de Marina Armada de México en el sup. Of. \# c0219/4345/13 del 5 de septiembre del 2013.

5 Todos los algoritmos son explicados en el acápite "Modelo de medición del poder naval". 
destacar que los indicadores seleccionados cumplen principalmente con tres requisitos, a saber: ser válidos, confiables y universales.

Para verificar su validez se revisan, cuidadosamente, las dimensiones del poder naval consideradas por la publicación internacional IHS Jane's Fighting Ships 2013-2014 (Saunders S. 2013), la enciclopedia naval ilustrada norteamericana, editada por el Departamento de Estado de los Estados Unidos de América, la Guía del Poder Naval en el Mundo (Wragg 2012), los textos especializados de Camil Busquets (Vilanova C. B. 2012) y Octavio Diez (2006) y la Guía de Barcos de Guerra actualizada de Chris Chant (2006), principalmente.

La confiabilidad fue establecida a partir de una revisión de la objetividad de la institución o autor de los textos revisados que contemplan a los indicadores del poder naval; y la universalidad, por su parte, se determina evaluando el uso de cada indicador en función de si se emplea a nivel mundial, regional o local.

Una vez seleccionados los indicadores, resulta necesario fijar los valores de ponderación de cada factor. Aquí, se debe establecer la medida estándar óptima de cada indicador evaluado, para que las medidas de los distintos países se comparen con el estándar óptimo y así obtener el valor del índice del poder naval; valor obtenido que se correlaciona con los índices del poder naval total de los otros países evaluados.

El modelo generado para la simulación de los resultados es un modelo de ecuaciones lineales, seleccionado siguiendo la propuesta de Heckbert et al. (2010, citado por Cardoso 2011: 7), considerando que no se contempla una retroalimentación dinámica de los datos. Las dimensiones establecidas como más adecuadas en la construcción del modelo para las variables independientes Capacidad Naval actual (CNa) y Capacidad Naval potencial (CNp) son citadas a continuación y están en negritas (Cuervo Vázquez 2014: 15-17).

Poder Naval actual (PNa): TB: Tipo de Buque (Pa: portaaviones, ph: portahelicópteros, cp: cruceros pesados, cr: cruceros, subm: submarinos misilísticos, subc: submarinos crucero, suba: submarinos de ataque, Dd: destructores, fr: fragatas, $\mathrm{Cb}$ : corbetas, an: anfibios, min: minadores, $\mathrm{dr}$ :

dragaminas/cazaminas, Pt: patrulleros); Dz: Desplazamiento (Se mide en toneladas); Es: Eslora (Se mide en metros); Pr: Propulsión (nu: Nuclear, nnu, No nuclear, dia: Doble/alternativa), Arm: Armamento (mb: Misil balístico, mcr: Misil crucero, mss: Misil superficie-superficie, msa: Misil superficie-aire, mssub: Misil superficie-submarino, tor: Torpedos, ame: Ametralladoras, min: Minas, car: Cargas de profundidad); V: Velocidad (Se mide en nudos); Aem: Aeronaves embarcadas; \#Aem: Número de aeronaves embarcadas; \#Me: Número de misiles embarcados (\#Mb: Misil balístico, \#Mcr: Misil crucero, \#Mss: Misil superficie-superficie, \#Msa: Misil superficie-aire, \#mssub: Misil superficie-submarino); \#Us: número de unidades de superficie (Se mide en números reales); Sa: Sistemas de armas (Aeg: Aegis/similar, Par: Parcial, Gss: Guerra superficie-superficie, Gsa: Guerra superficie-aire, Gssub: Guerra superficie-submarino); CNp: Capacidad Naval potencial, per: Personal (Se mide en número de efectivos encuadrados
Hacia una medición del poder naval en América Latina

Noé Cuervo Vázquez Marcos Pablo Moloeznik 
en el servicio activo); Pib: Producto interno bruto (Se mide en miles de dólares); Ri: Reservas internacionales (Se mide en millones de dólares); Ip: Infraestructura portuaria (Índice de infraestructura portuaria); Im: Infantería de Marina (Si existe el cuerpo de Infantería de Marina); Fz: Fuerzas Navales (Se mide la cantidad de Fuerzas o Flotas navales); Enf: Educación naval de formación (Si existe una escuela naval de formación de oficiales); Enp: Educación naval de posgrado (Si existe una escuela naval de nivel de posgrado); Dtn: Desarrollo tecnológico naval (Si existe un centro de desarrollo tecnológico naval); Ane: Aeronaves no embarcadas.

Una vez identificadas las dimensiones y sus indicadores, es necesario determinar el valor de ponderación por cada dimensión. Para ello, se aplica una encuesta dirigida a informantes clave de una población que cuente con información relevante del Poder Naval, que proporcione el nivel de importancia de cada dimensión y de cada indicador dentro de la valoración del Poder Naval de un Estado. Como población se determinó al personal de alumnos colegiados del Centro de Estudios Superiores Navales (CESNAV) de la Secretaría de Marina/Armada de México, habiendo seleccionado una muestra representativa de 37 oficiales superiores y jefes, con los grados de Capitanes de Navío, Capitanes de Fragata, Capitanes de Corbeta, así como de invitados, pertenecientes a la Maestrías de Seguridad Nacional y del Diplomado de Estado Mayor.

Con el instrumento ya construido, se distribuyó y se les solicitó que enumeraran, de acuerdo al orden de importancia (siendo el número uno el más importante), las dimensiones y los indicadores de cada reactivo, obteniéndose los siguientes resultados:

Tabla 1.

Valores ponderados Capacidades Navales actua

\begin{tabular}{clcl}
\hline $\begin{array}{c}\text { Nivel de } \\
\text { importancia }\end{array}$ & \multicolumn{1}{c}{ Dimensión } & $\begin{array}{c}\text { Valor de } \\
\text { ponderación }\end{array}$ & Orden $p^{n}$ \\
\hline 1 & Tipo de Buque & 1 & $P 1$ \\
\hline 1 & Número de Buques & 1 & $P 3$ \\
\hline 3 & Sistema de Armas & .830 & $P 2$ \\
\hline 4 & Número de misiles embarcados & .747 & $P 4$ \\
\hline 4 & Aeronaves embarcadas & .747 & $P 5$ \\
\hline 6 & Número de aeronaves embarcadas & .581 & $P 11$ \\
\hline 8 & Armamento & .415 & $P 10$ \\
\hline 10 & Velocidad & .249 & $P 9$ \\
\hline 10 & Eslora & .249 & $P 7$ \\
\hline 12 & Propulsión & .083 & $P 8$ \\
\hline 12 & Desplazamiento & .083 & $P 6$ \\
\hline
\end{tabular}

Fuente: elaboración propia. 
En tanto que, para poder construir un algoritmo que mida al poder naval, se debe entender que la sostenibilidad de las Capacidades actuales del Poder Naval debe ser entendida como un problema complejo que presenta relaciones no lineales entre todos los factores que lo componen. En términos intuitivos significa, por ejemplo, que mayores niveles de esfuerzos navales (cantidad de embarcaciones principalmente), no tienen necesariamente mayores beneficios en el Poder Naval de un Estado.

Si bien no interesa caer en tecnicismos matemáticos, hay que tener presente algunas nociones básicas del proceso de modelación, para facilitar la comunicación del modelo que se propone (Cuervo Vázquez 2014: 15-17). Es decir, la comprensión del planteamiento del problema y las simulaciones computacionales requieren al menos un manejo intuitivo del funcionamiento del proceso de modelación matemática.

Así, en la figura 1 se muestra cómo quedaron relacionados los factores correspondientes a las Capacidades Navales actuales para poder encontrar su relación matemática.

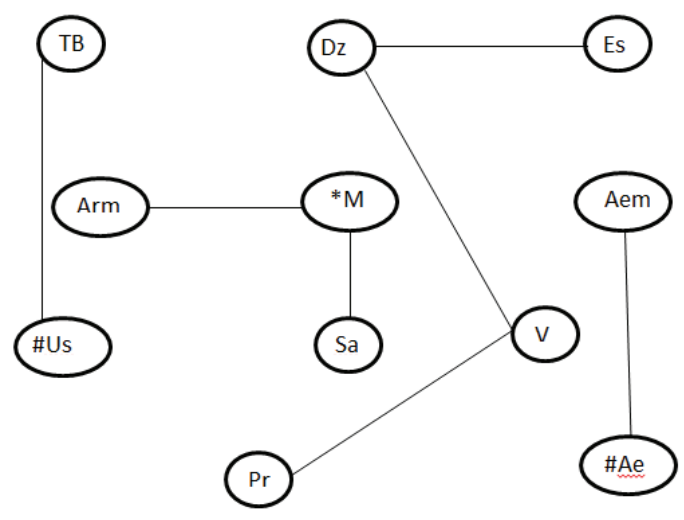

Fuente: elaboración propia.

Bajo los resultados de la figura anterior, se puede observar que se forman cuatro conjuntos con características similares en relación directa:

- Conjunto 1: Unidad de superficie, que relaciona al Tipo de Buque (TB) con la cantidad de unidades de superficie (\#Us) de manera directa, por lo que se consideró multiplicar ambos factores.

- Conjunto 2: Capacidad de Fuego, que relaciona al Armamento (Arm) con los Sistemas de armas (Sa) y con el número de Mísiles (\#M). Aquí también se consideró efectuar una multiplicación entre los tres factores relacionados.

- Conjunto 3: Aeronaves, que relaciona al factor Aeronaves embarcadas (Ae) con el factor Número de aeronaves embarcadas (\#Aem), factores que también fueron multiplicados.
Hacia una medición del poder naval en América Latina

Noé Cuervo Vázquez Marcos Pablo Moloeznik

Figura 1.

Relación de las Capacidades Navales actuales 
- Por último, el Conjunto 4: Características del Buque, contempla cuatro factores, tres de los cuales, Eslora (Es), Desplazamiento (Dz) y Propulsión (Pr) se consideró que debían ser solo sumados y su resultado multiplicarlo por el factor Velocidad (V).

Tomando en consideración los resultados obtenidos tanto en la encuesta (que permitió obtener los valores de las ponderaciones "p" y "q") y de las relaciones que definieron los conjuntos anteriores, el algoritmo resultante para obtener el valor de la variable: Capacidad Naval actual (CNa), es el siguiente:

$$
\begin{gathered}
C N a=\sum_{1}^{n}\left[\left(\left(T B p_{1} q_{1} * \# U_{s} p_{3}\right)+\left(S_{a} p_{2} q_{2} * \# M_{\theta} p_{4} * A_{r m} p_{10} q_{5}\right)+\left(A_{\theta m 2} p_{5} * \# A_{\theta m} p_{11}\right)+\right.\right. \\
\left.\left.+\left(\frac{D_{z}}{D_{z \text { Bass }}} p_{6}+\frac{E_{s}}{E_{s \text { Base }}} p_{7}+P_{r} p_{8} q_{3}\right) * V p_{9} q_{4}\right)\right]\left(\frac{100}{C N_{a \text { Base }}}\right)
\end{gathered}
$$

En donde las dimensiones de la variable $\mathrm{CNa}$ se multiplican por sus valores ponderados ( $p$ y $q$ ) y el producto total se multiplica por cien y se divide entre el valor de la variable $\mathrm{CNa}$ del país base del modelo, que para este trabajo de investigación es el valor del Poder Naval de los Estados Unidos de América.

En cuanto a la segunda variable independiente CNp, se puede apreciar el siguiente orden de importancia, resultado de la encuesta realizada a expertos

\begin{tabular}{|c|c|c|c|}
\hline $\begin{array}{l}\text { Nivel de } \\
\text { importancia }\end{array}$ & Dimensión & Valor de ponderación " $p$ " & Orden " $p$ " \\
\hline 1 & Producto Interno Bruto & 1 & $P 1$ \\
\hline 1 & Flota Naval & 1 & $P 2$ \\
\hline 1 & $\begin{array}{l}\text { Desarrollo Tecnológico } \\
\text { Naval }\end{array}$ & 1 & $P 3$ \\
\hline 2 & Infraestructura portuaria & 0.88 & P4 \\
\hline 4 & Personal & 0.66 & P5 \\
\hline 7 & Infantería de Marina & 0.33 & P6 \\
\hline 8 & $\begin{array}{l}\text { Educación Naval de } \\
\text { Formación }\end{array}$ & 0.22 & $P 7$ \\
\hline 9 & $\begin{array}{l}\text { Educación Naval de } \\
\text { Postgrado }\end{array}$ & 0.11 & P8 \\
\hline 9 & Reservas Internacionales & 0.11 & $P 9$ \\
\hline 12 & Aeronaves NO embarcadas & 0.083 & P10 \\
\hline
\end{tabular}
en el tema:

Fuente: elaboración propia. 
Tomando en consideración los resultados obtenidos, se analizó que la relación entre los factores de las Capacidades Navales potenciales no tenían una relación entre ellos que obligara a realizar alguna multiplicación, y todo quedó reducido a la sumatoria de los citados factores.

Sin embargo, fue necesario establecer una constante que nos aproximara a un valor más real de esta variable. Por ello, se consideró que el Producto Interno Bruto (Pib), las Flotas Navales (Fz) y el Desarrollo Tecnológico (Dtn) son los factores más significativos y debían multiplicarse por dos; el factor Infraestructura Portuaria (Ip) se multiplica por la constante 1.5 y el factor Personal (Per) por la constante 1.3; el resto de los factores no son multiplicados por constante alguna y sólo se multiplican al igual que los demás factores por sus ponderaciones "p" $y$ " $q$ ".

El algoritmo resultante para obtener el valor de la Capacidad Naval potencial (CNp), es:

$$
\begin{aligned}
& C N p=\left((2)\left(\frac{p_{t b}}{p_{t b \text { BaSS }}} p_{1}\right)+(2)\left(\frac{f_{z}}{f_{z B A S S}} p_{2}\right)+(2)\left(\frac{d_{C M}}{d_{C M B A S S}} p_{3}\right)+(1.5)\left(\frac{i_{y}}{i_{W B A S S}} p_{4}\right)+\right. \\
& \left.+(1.3)\left(\frac{P_{\text {srr }}}{P_{\text {ar Bass }}} p_{5}\right)+\frac{i_{m}}{i_{m \text { Bass }}} p_{6}+\theta_{n f} p_{7}+\theta_{n p} p_{8}+\left(\frac{r_{t}}{r_{1 \text { Bass }}}\right) p_{9}+A_{n e} p_{10}\right)
\end{aligned}
$$

En ambas variables independientes las consonantes "p" y "q" se refieren al porcentaje de ponderación tanto a nivel horizontal (que corresponde a la ponderación dentro de la variable y a la ponderación dentro de la propia dimensión).

Una vez obtenidos los valores de las variables independientes, el algoritmo final para obtener el valor de la variable dependiente (Poder Naval total), quedó de la siguiente manera:

$$
P N t=\frac{C N_{Q}+C N_{p}}{2}
$$

Con los valores correspondientes a las variables independientes y la variable dependiente, se integra un nuevo algoritmo, con el fin de determinar un índice de riqueza marítima nacional que permitiera interactuar con los valores obtenidos y encontrar cómo se ve reflejado el Poder Naval de un Estado con las riquezas marítimas que debe proteger. Para ello, y de acuerdo al marco teórico propuesto, se establece que el índice de riqueza marítima nacional (IRMN) ${ }^{6}$ está en función de los siguientes factores (Cuervo Vázquez 2014: 22): pc: plataforma continental (se mide en kilómetros cuadrados),

6 Este índice de riqueza marítima nacional no abarca todos los intereses marítimos de un Estado, por ser tema del Poder Marítimo Nacional, el cual no es abordado en la presente contribución. Sin embargo, los factores considerados permiten interactuar con un nivel de confianza aceptable a la variable obtenida del Poder Naval total de un país y determinar el nivel de protección de la citada riqueza.
Hacia una medición del poder naval en América Latina

Noé Cuervo Vázquez Marcos Pablo Moloeznik 
zp: zonas de pesca (se mide en kilómetros cuadrados), Aa: Área arrecifal (se mide en kilómetros cuadrados), zee: zona económica exclusiva (se mide en kilómetros cuadrados), ep: exportaciones petroleras (se mide en barriles diarios), lpi: Índice de eficiencia logística (se mide según el Banco Mundial), tmc: tráfico marítimo de contenedores (se mide en número de contenedores), ipib: $\%$ importaciones del PIB (se mide en porcentaje), epib: \% exportaciones del PIB (se mide en porcentaje).

El algoritmo para obtener el IRMN ${ }^{7}$ que se utiliza en el presente modelo quedó sujeto a una sumatoria de todos los factores multiplicados por su valor de ponderación " $p$ ", de la siguiente manera:

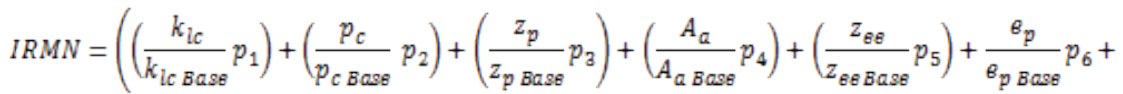

$$
\begin{aligned}
& \left.+\left(\frac{l_{p i}}{l_{\text {pi Bass }}} p_{1}\right)+\left(\frac{t_{m c}}{t_{m c \text { Bass }}} p_{1}\right)+\left(\frac{i_{\text {pib }}}{i_{\text {pib Bass }}}\right) p_{9}+\left(\frac{\varepsilon_{\text {pib }}}{\varepsilon_{\text {pib Bass }}} p_{1}\right)\right)
\end{aligned}
$$

Una vez con el valor del IRMN, se procedió a obtener el índice de defensa marítima (IDM), el cual es el resultado de multiplicar el Poder Naval total por 100 y dividirlo entre el IRMN; la diferencia del IDM con 100 nos dará el porcentaje de riqueza marítima sin protección (\%RSP). Estos valores para la presente contribución son indicadores de cobertura del Estado ante actores no estatales; para obtener un comparativo entre naciones, es necesario comparar el Poder Naval total (PNt) de cada país.

$$
I D M=\frac{P N_{t} * 100}{I R M N} \quad \% R S P=100-I D M
$$

El modelo concluye asignando el tipo de armada que su poder naval total le otorga, según la siguiente propuesta de nueve niveles de Till (2007: 153-154): - Armada de proyección de fuerza global mayor - completa (ejemplo: EE.UU.).

Puede trasladar su poder naval a cualquier parte del mundo y en varios escenarios a la vez de manera permanente.

- Armada de proyección de fuerza global mayor-parcial (ejemplo: extinta Unión de Repúblicas Socialistas Soviéticas).

Puede trasladar su poder naval a cualquier parte del mundo en máximo dos escenarios a la vez de manera temporal.

- Armada de proyección de fuerza global mediana (ejemplo: Francia, Gran Bretaña).

7 El IRMN, es un índice de la riqueza marítima de un país con relación a valores base obtenidos de los primeros puestos de los reportes publicados, donde se localizan los indicadores seleccionados. 
Puede trasladar una parte de su poder naval a cualquier parte del mundo en un escenario a la vez de manera temporal.

- Armada de proyección de fuerza regional mediana (ejemplo: India, China, Japón).

Puede trasladar su poder naval a escenarios cercanos a su territorio dentro de la región geográfica que ocupa.

- Armada de proyección de fuerza adyacente (ejemplo: Portugal, Israel, Sudáfrica).

Puede proyectar su poder naval a las áreas marítimas adyacentes a su zona económica exclusiva (ZEE).

- Armada de defensa territorial marítima (ejemplo: Noruega, Egipto).

Tiene la capacidad de negar el uso del mar al enemigo y presentar combate sólo en la ZEE.

- Armada de defensa territorial costera (ejemplo: Omán, Singapur).

Tiene la capacidad de negar el uso del mar al enemigo y presentar combate sólo en el mar territorial.

- Armada de vigilancia policial (ejemplo: México, Sri Lanka).

Tiene la capacidad de mantener el Estado de derecho en su ZEE contra la delincuencia organizada, pero no tiene la capacidad de negar el uso del mar a la fuerza naval de otro Estado.

- Armadas simbólicas.

No tiene la capacidad de mantener el Estado de derecho en su ZEE contra la delincuencia organizada.

\section{Una aproximación a la medición del poder naval en América Latina}

El continente americano cuenta con 35 países divididos principalmente en dos regiones, una conformada por los Estados Unidos de América y Canadá y otra que integra a los países latinoamericanos. ${ }^{8}$ En la presente tesis, se realizó un análisis de las Armadas nacionales de los 33 países que integran la región de Latinoamérica, ${ }^{9}$ divididos, a su vez, en cuatro áreas:

Cabe señalar que se hace una presentación de las armadas de los países latinoamericanos a partir de la consideración que no se tomaron en cuenta los buques de porte menor a 200 toneladas de desplazamiento, con excepción de las seis patrullas costeras clase Constitución de Venezuela, que a pesar de tener

8 Esta división tradicional se utiliza en el presente trabajo, en el que se consideraron como parte de Latinoamérica, a los países del Caribe y Sudamérica que no son hispano-parlantes, pero que por sus condiciones de países en vías de menor grado de desarrollo relativo con respecto al centro desarrollado, tienen características similares como nación.

9 Los datos de las Armadas, se basan sólo en fuentes abiertas especializadas, principalmente la información que contiene el Jane's Fighting Ships 2011-2012, The World Sea Power Guide del 2012 y Barcos de Guerra (Chant 2006).
Hacia una medición del poder naval en América Latina

Noé Cuervo Vázquez Marcos Pablo Moloeznik 


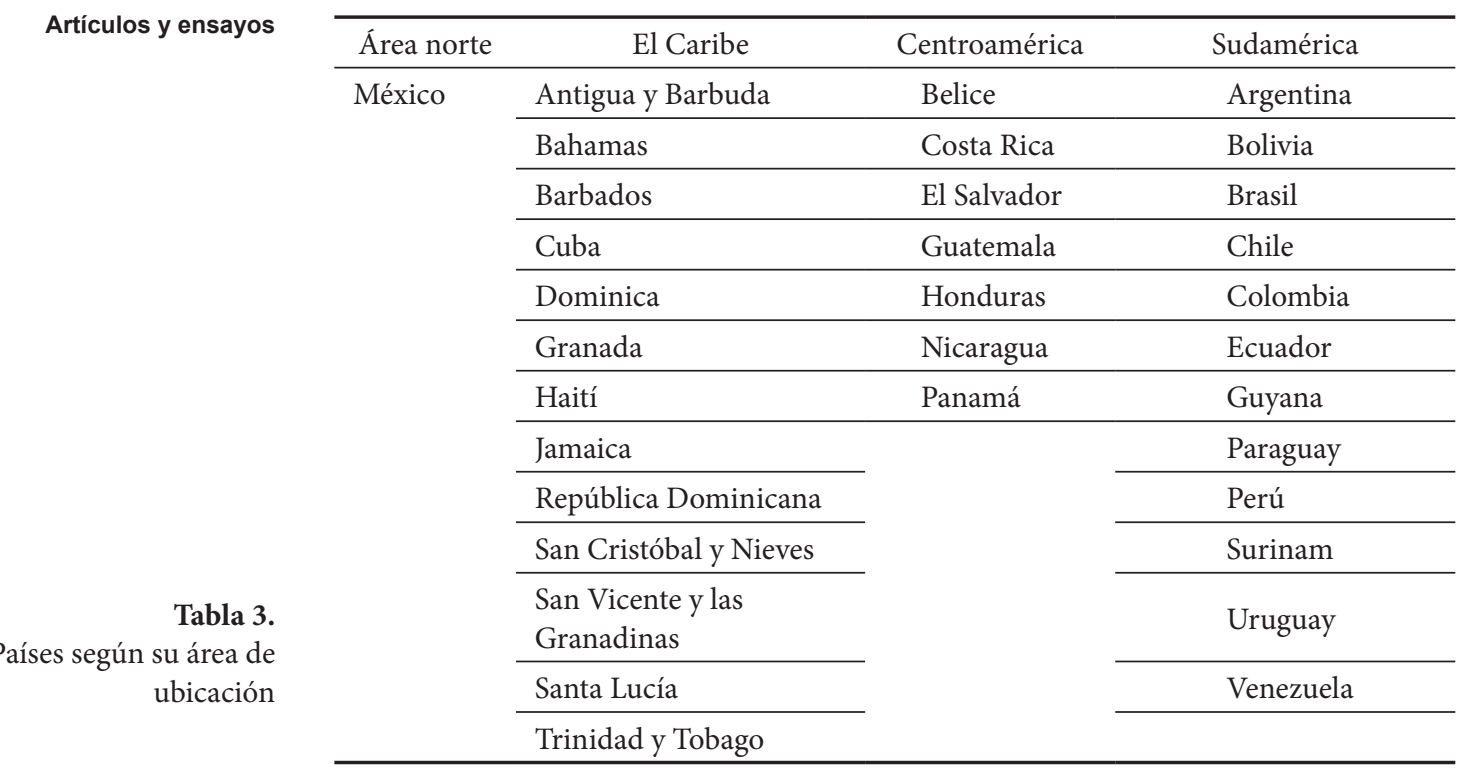

Fuente: elaboración propia.

un desplazamiento de 173 toneladas, sus misiles superficie-superficie (SSM) les confieren un importante poder de fuego.

Tratándose del área del Caribe, de los 13 países que la integran, Jamaica cuenta con dos patrullas costeras, Trinidad y Tobago con tres patrullas costeras y República Dominicana con dos patrullas costeras, sin embargo, tienen un valor de su poder naval menor a la unidad, por lo que no requieren que se les analice mayormente. Cuba, por su parte, cuenta con un buque de la clase Pauk II artillado con seis misiles superficie-aire (SAM) SA-N-5, una patrulla costera clase Trawler con 46 misiles SSM y seis patrullas costeras clase OSA II, también artillada con 46 SSM, ambas con misiles SS-N-2B, así como con cuatro unidades de superficie más. El resto de los países del Caribe no tienen unidad de superficie alguna que desplace el tonelaje requerido.

En Centroamérica, solamente dos países cuentan con unidades de superficie a considerar: Panamá que tiene dos patrullas costeras y El Salvador que cuenta con una. Sin embargo, su índice de poder naval, junto con el resto de los países de esa área, no resultó significativo para la presente contribución.

En Sudamérica la situación es diferente. Bolivia, Guyana y Surinam no cuentan con buques de porte mayor a 200 toneladas y su nivel de poder naval tiende a cero. Uruguay, por su parte, cuenta con dos fragatas clase Joao Belo, una patrulla oceánica clase Wangerooge, tres patrullas clase Kondor II y dos patrullas clase Vigilante, todas sin capacidad de misiles.

Paraguay también cuenta con una armada muy pequeña, con cinco buques divididos de la siguiente manera: todos son buques patrulla, uno de la clase 
River Defense, dos de la clase Bouchard, uno de la clase Itaipu y uno de la clase River Patrol. Al igual que Uruguay, su armada no cuenta con capacidad de misiles.

Los países restantes de Sudamérica cuentan con armadas más estructuradas y mejor equilibradas, algunas, como Brasil, con portaaviones y la mayoría con fuerza submarina.

Argentina tiene $1084386 \mathrm{~km}^{2}$ de Zona Económica Exclusiva (ZEE), $5029 \mathrm{~km}$ lineales de costa, $785879 \mathrm{~km}^{2}$ de plataforma continental, $166486 \mathrm{~km}^{2}$ de zonas pesqueras, un tráfico de contenedores de 2136911 TEU, ${ }^{10}$ un índice de infraestructura portuaria del 3.7 y una exportación petrolera de 238100 barriles de crudo diarios, como parte de sus riquezas marítimas. Para su protección, la Armada Argentina (ARA) cuenta con 18249 elementos, de los cuales 2800 están destinados a la Infantería de Marina y 2000 a la Aviación Naval. Tiene una pequeña fuerza submarina de construcción alemana integrada con un submarino de ataque de la clase Salta y dos de la clase Santa Cruz y tiene planes de construir un submarino de propulsión nuclear (Wragg 2012: 254). ${ }^{11}$ Además, cuenta con cuatro destructores de la clase Meko 360, artillados con 70 SSM Exocet MM40, 13 SAM Aspide y espacio para un helicóptero Fennec; tres fragatas clase Drummond, con 42 SSM Exocet MM38, seis fragatas clase Meko 140 con 42 SSM Exocet MM38; dos patrullas clase Intrépida, con 42 SSM Exocet MM38 y 15 buques patrullas de desplazamiento mayor a 200 toneladas.

Brasil tiene una ZEE de $3179693 \mathrm{~km}^{2}, 7491 \mathrm{~km}$ lineales de costa, $708805 \mathrm{~km}^{2}$ de plataforma continental, $376117 \mathrm{~km}^{2}$ de zonas pesqueras, un tráfico de contenedores de 8649821 TEU, un índice de infraestructura portuaria de 2.7 y una exportación petrolera de 801200 barriles de crudo diarios como parte de sus riquezas marítimas. La Marina de Guerra de Brasil tiene 38 800 elementos de los cuales 15800 son infantes de marina, siendo la única del subcontinente -y unas de las diez del orbe- en contar con un portaaviones de la clase Clemenceau (São Paulo), con un ala embarcada de 15 aviones Mcdonnell Douglas Skyhawk, al que se suman cuatro submarinos de ataque de la clase Scorpene modificada, cuatro submarinos de ataque de la clase Tupi y un submarino de ataque de la clase Tikuna. Al igual que Argentina, existen planes para la construcción de un submarino de propulsión nuclear y ha habido informes no confirmados que la República Popular China está ayudando en el diseño a cambio de ayuda con el funcionamiento de arrastre de aviones de ala fija embarcados (Wragg 2012: 518). Su fuerza de misiles en unidades de superficie está distribuida en tres fragatas Broadsword con 42 SSM Exocet MM38 y cinco SAM

10 El tráfico portuario de contenedores mide el flujo de contenedores del modo de transporte terrestre a marítimo y viceversa, en unidades equivalentes a 20 pies (TEU), un contenedor de tamaño estándar.

11 Cabe destacar que esta afirmación hecha por Wragg (2012) parece muy aventurada y completamente descartada por Moloeznik y Guevara (2014).
Hacia una medición del poder naval en América Latina

Noé Cuervo Vázquez Marcos Pablo Moloeznik 
Sea Wolf, capaz de transportar dos helicópteros Súper Lynx ASW; seis fragatas Niteroi con 70 SSM Exocet MM40 y 21 SAM Aspide 2000, cuatro corbetas clase Inhauma con 70 SSM Exocet MM40 y una corbeta clase Barroso con 70 SSM Exocet MM40, capaz de embarcar un helicóptero Súper Lynx; cuenta, además, con 34 buques tipo patrullas, dos buques de guerra anfibia de la clase Thomaston y uno de la clase Newport, uno de la clase Sir Galand, uno de la clase Sir Bedivere, tres barcazas de desembarco de más de 200 toneladas de desplazamiento y seis buques tipo dragaminas de la clase Schutze. De acuerdo con Moloeznik (2016), el 1 de marzo de 2013, la entonces presidenta Dilma Rousseff inauguró la planta de fabricación de naves a propulsión nuclear. Con esto, Brasil ingresará al selecto grupo de países equipados con submarinos de propulsión nuclear y se convertirá en el sexto país en el mundo capaz de desarrollar ese tipo de submarino con la clara intención de proyectarse sin descuidar sus zonas marítimas. Tampoco debe soslayarse el reciente anuncio del ministro brasileño de defensa, Celso Amorim, quien afirmó que la construcción de un portaaviones propio figura entre los futuros proyectos del país.

Chile, por su parte, cuenta con $2009299 \mathrm{~km}^{2}$ de ZEE, $6435 \mathrm{~km}$ lineales de costa, $160916 \mathrm{~km}^{2}$ de plataforma continental, $211070 \mathrm{~km}^{2}$ de zonas pesqueras, un tráfico de contenedores de 3406267 TEU, un índice de infraestructura portuaria del 5.2 y una exportación petrolera de 52390 barriles de crudo diarios, como parte de sus riquezas marítimas. Mientras que para su protección, la Armada de Chile está conformada por 25699 elementos, de los cuales 807 son reclutas en entrenamiento voluntario de 22 meses. Tiene en servicio activo dos clases de submarinos, con dos unidades igualmente, la clase Scorpene y la clase Thomson; cuenta con dos fragatas clase Doorman con 130 SSM Harpoon y 15 SAM Sea sparrow, dos fragatas clase Latorre de construcción holandesa artilladas con 130 SSM Harpoon y 46 SAM SM-1MR, tres fragatas de la clase Leander con 130 SSM Harpoon y 6 SAM Sea wofl, una fragata Clase Broadword con 130 SSM Harpoon y 10 SAM Barak I y dos buques patrulla clase SAAR 4 armadas con 36 SSM Gabriel II. Tiene, además, tres clases de buques tipo patrulla: cuatro clase Tiger (Riquelme), dos clase Piloto Pardo y seis clase Micalvi; dos buques de guerra anfibia de la clase Batral (Maipo), uno de la clase Newport, uno de la clase Alvsborg y dos de la clase Elicura.

Colombia tiene $817816 \mathrm{~km}^{2}$ de ZEE en dos litorales, $3208 \mathrm{~km}$ lineales de costa, $43316 \mathrm{~km}^{2}$ de plataforma continental, $36460 \mathrm{~km}^{2}$ de zonas pesqueras, un tráfico de contenedores de 2572012 TEU, un índice de infraestructura portuaria de 3.5 y una exportación petrolera de 400700 barriles de crudo diarios. Por su parte, la Armada colombiana tiene en servicio activo a 21000 elementos, de los cuales 7200 son conscriptos, 146 son de aviación naval y 9000 son infantes de marina. Tiene también dos submarinos de ataque de la clase Pijao, cuatro fragatas clase Alm. Padilla con 70 SSM Exocet MM40 (siendo las únicas con misiles embarcados con que cuenta) con capacidad para un helicóptero ligero como un Fennec o un Bo-105, dos buques de guerra de minas, 18 buques patrullas y siete barcazas de desembarco de la clase Morrosquillo. 
Ecuador es poseedor de una ZEE de $236597 \mathrm{~km}^{2}$, de $2237 \mathrm{~km}$ lineales de costa, $24679 \mathrm{~km}^{2}$ de plataforma continental, $23894 \mathrm{~km}^{2}$ de zonas pesqueras, un tráfico de contenedores de 1291494 TEU, un índice de infraestructura portuaria de 4.2 y una exportación petrolera de 333400 barriles de crudo diarios. Su Armada es de 7283 efectivos encuadrados, incluyendo a 2160 infantes de marina y 375 de aviación naval. Tiene dos submarinos de ataque la clase Shyri, dos fragatas clase Leander con 70 SSM Exocet MM40 y 5 SAM Sea cat con capacidad para un helicóptero Bell Jet Ranger, seis corbetas de la clase Esmeralda con plataforma de anaveaje, armadas con 70 SSM Exocet MM40 y 13 SAM Aspide, tres buques patrulla de la clase Lurssen y 42 SSM Exocet MM40. Cuenta además con seis buques patrullas sin misiles clases Cherokee (1), Espada (2) e Isla Fernandina (3).

Perú es un país andino con litoral en el Pacífico, tiene $906454 \mathrm{~km}^{2}$ de ZEE, $2997 \mathrm{~km}$ lineales de costa, $79198 \mathrm{~km}^{2}$ de plataforma continental, $55339 \mathrm{~km}^{2}$ de zonas pesqueras, un tráfico de contenedores de 1621484 TEU, un índice de infraestructura portuaria de 4.7 y una exportación petrolera de 73280 barriles de crudo diarios. La marina de guerra de Perú está compuesta por 23246 elementos, incluyendo 3500 infantes de marina, 1000 miembros de la guardia costera y 800 de aviación naval. Tiene seis submarinos de ataque clase Angamos, un crucero de batalla (el único en Latinoamérica) clase De ruyter armado con 160 SSM Otomat Mk2, cuatro fragatas clase Lupo modificada con 160 SSM Otomat Mk2 y 13 SAM Aspide, cuatro fragatas Lupo (Aguirre) artilladas con 160 misiles SSM Otomat Mk2 y 13 SAM Aspide con capacidad para un helicóptero Bell 212, seis buques patrulla clase Velarde con 42 SSM, 11 buques patrullas sin capacidad de misiles y cuatro buques de guerra anfibia de la clase Terrebone Parrish.

El último país a analizar del área de Sudamérica es la República Bolivariana de Venezuela, la cual cuenta con una ZEE de $471507 \mathrm{~km}^{2}, 4800 \mathrm{~km}$ lineales de costa, $99889 \mathrm{~km}^{2}$ de plataforma continental, $109426 \mathrm{~km}^{2}$ de zonas pesqueras, un tráfico de contenedores de 1296419 TEU, un índice de infraestructura portuaria de 2.5 y una exportación petrolera de 1871000 barriles de crudo diarios. Su marina de guerra está compuesta por 17994 elementos que incluyen a 3200 conscriptos que prestan servicio hasta por 30 meses, aunque el servicio militar obligatorio es por selección y el periodo es variable; tiene 7000 infantes de marina y 500 en el cuerpo de aviación naval. Sus barcos han sido adquiridos de diferentes países, incluyendo a Corea de Sur, pero el país se ha ido moviendo hacia la izquierda en el ámbito político y ha sido la primera nación latinoamericana en realizar ejercicios navales con la Armada Rusa, cuando los barcos de la Flota del Norte visitaron el Caribe en el 2008. Es probable que en el futuro, la Armada Bolivariana de Venezuela se acerque más a Rusia e incluso a la Armada China (Wragg 2012), aunque, de acuerdo con Moloeznik (2016), el gobierno se está acercando a los chinos para obtener suministros, logística y equipo terrestre (blindados y artillería) para la infantería de marina, pero la armada apuesta por la obtención de equipo eu-
Hacia una medición del poder naval en América Latina

Noé Cuervo Vázquez Marcos Pablo Moloeznik 
ropeo únicamente. Cuenta con dos submarinos de ataque de la clase Sabalo y 61,2 mts. de eslora, seis fragatas clase Lupo modificada 160 SSM Otomat Mk2 y 13 SAM Aspide con capacidad para un helicóptero AB-212 ASW, seis patrullas costeras clase Constitución con 80 SSM Otomat Mk2. Estos son los únicos buques que tienen capacidad de lanzamiento de misiles. Tiene, además, cuatro buques patrullas de la clase Alligator, cuatro buques patrulla clase Guaiqueri, cuatro patrullas clase Guaicamacuto, dos barcazas de guerra anfibia clase Ajeera y un buque logístico auxiliar de la clase AORH.

México es el último país de la región (aunque del área norte) que cuenta con una Armada estructurada. Tiene una ZEE de $3269386 \mathrm{~km}^{2}$ de ZEE en dos litorales, $11953 \mathrm{~km}$ lineales de costa, $402064 \mathrm{~km}^{2}$ de plataforma continental, $251122 \mathrm{~km}^{2}$ de zonas pesqueras, un tráfico de contenedores de 3893 $946 \mathrm{TEU}$, un índice de infraestructura portuaria de 4.4 y una exportación petrolera de 1511000 barriles de crudo diarios. Su fuerza naval está constituida por 56324 elementos que incluyen a 18000 infantes de marina y 1250 en el cuerpo de aviación naval. Como unidades de superficie tiene un destructor clase Gearing Fram I, sin capacidad de misiles, cuatro fragatas clase Knox, con un lanzador octuple Mk 25 para SAM Sea Sparrow, dos fragatas clase Bronstein, dos buques patrullas clase SAAR 4.5 con 36 SSM Gabriel y 10 SAM Barak I, y los siguientes buques tipo patrulla oceánica sin capacidad de misiles: cuatro de la clase Holzinger, cuatro de la clase Durango, tres de la clase Sierra, seis de la clase Oaxaca, seis de la clase Uribe, dos de la clase Demócrata y 10 de la clase AUK. Además cuenta con dos buques de guerra anfibia de la clase Newport y dos buques de guerra anfibia de la clase Montes Azules. Los buques antes mencionados, con excepción de los clase AUK, tienen plataforma de anaveaje y su aviación de ala móvil tiene incorporados helicópteros Phanter, Black Hawk, Bolkow, Fennec, MI-17 y MD.

Recapitulando, el modelo de medición utiliza las variables descritas para obtener los valores de los siguientes índices: Capacidades Navales actuales, Capacidades Navales potenciales, Poder Naval Total, Tipo de Armada, Índice de Riqueza Marítima Nacional, Índice de Defensa Marítima Policial, Índice de Defensa Naval, Porcentaje de Riqueza Marítima sin protección actual (contra infractores de la ley), Porcentaje de Riqueza Marítima sin protección actual (contra otra Fuerza Naval).

Dentro del primer rubro Capacidades Navales actuales, se observa que Chile es el país que actualmente tiene el mayor índice de esta categoría. Su poder de fuego obtenido gracias a sus fragatas clase Doorman, Latorre y Leander con sus misiles Harpoon ha sido determinante para alcanzar el primer puesto por encima de las demás naciones.

En segundo lugar, se encuentra Perú teniendo como buques más representativos a sus ocho fragatas Lupo (4 modificadas y 4 "Aguirre") con sus misiles Otomat MK2.

Brasil, a pesar de ser el único país con portaaviones de la región, no cuenta con una capacidad de misiles representativa, y por esa razón sus capacidades 
navales actuales lo colocaron solo en el tercer puesto. De hecho, al ser su ala embarcada en el portaaviones São Paulo un tanto antigua, los buques que más incrementaron su índice de Capacidades Navales actuales fueron sus seis fragatas Niteroi con sus misiles Exocet MM40.

En cuarto lugar se colocó Venezuela con sus seis fragatas Lupo modificadas y sus misiles Otomat MK2. Casi con las mismas capacidades navales se encuentra Ecuador, teniendo como buques más importantes a sus seis corbetas clase Esmeralda artilladas con misiles Exocet MM40.

Argentina le sigue en capacidades navales actuales con sus cuatro fragatas MEKO 360 y sus misiles Exocet MM40, mientras que Colombia, Cuba y México son los últimos tres países de los nueve analizados, que el modelo coloca con las capacidades navales actuales más bajas (obviamente, sin considerar a los países del Caribe, Centroamérica y Sudamérica, que no cuentan con armadas significativas). El modelo indica que los buques que aportan más al índice de capacidades actuales a Colombia, son sus cuatro fragatas clase Alm. Padilla con 70 SSM Exocet MM40, a Cuba son sus seis patrullas costeras clase OSA II artilladas con misiles SS-N-2B y a México son sus dos buques patrullas clase SAAR 4.5 con misiles Gabriel. (Cuervo Vázquez 2014: 33-34)

\begin{tabular}{lc}
\hline PAÍS & Capacidades actuales \\
\hline Chile & 23,95 \\
\hline Perú & 17,1 \\
\hline Brasil & 9,87 \\
\hline Venezuela & 6,42 \\
\hline Ecuador & 6,32 \\
\hline Argentina & 4,94 \\
\hline Colombia & 2,72 \\
\hline Cuba & 1,63 \\
\hline México & 1,38 \\
\hline
\end{tabular}

Fuente: elaboración propia. ${ }^{12}$

Con relación a sus Capacidades Navales potenciales, el país que tiene en estos momentos el mayor potencial de desarrollo es México, basado principalmente en la cantidad de elementos en servicio activo junto a la cantidad de infantes de marina, que supera a todos sus vecinos, aunado a la calidad de su infraestructura portuaria. Sus valores analizados en el modelo de medición

12 Todas las figuras que se presentan, toman en consideración, por una parte, a un país de Latinoamérica y, por otra, el valor de un índice obtenido por medio del modelo de medición del Poder Naval descrito.
Hacia una medición del poder naval en América Latina

Noé Cuervo Vázquez Marcos Pablo Moloeznik

Figura 4.

Capacidades Navales actuales 
del Poder Naval, lo ubicaron escasamente por encima de Brasil, ya que ambos tienen importantes Productos Internos Brutos y Reservas Internacionales, y muy por encima de naciones como Colombia, Argentina, Venezuela y, sobre todo, Cuba. Los valores que se obtuvieron se indican en la figura 5.

Figura 5.

Capacidades Navales potenciales

\begin{tabular}{lc}
\hline PAÍS & Capacidades potenciales \\
\hline México & 23,95 \\
\hline Brasil & 17,1 \\
\hline Chile & 9,87 \\
\hline Perú & 6,42 \\
\hline Ecuador & 6,32 \\
\hline Colombia & 4,94 \\
\hline Argentina & 2,72 \\
\hline Venezuela & 1,63 \\
\hline Cuba & 1,38 \\
\hline
\end{tabular}

Fuente: elaboración propia.

La suma algebraica de las anteriores variables (Variables Independientes del presente trabajo), arrojan el siguiente valor de la variable dependiente Poder Naval total: el país con el mayor Poder Naval total en Latinoamérica es Chile, seguido de cerca por Perú y Brasil. Este Poder está basado en su importante arsenal de misiles y sus sistemas de armas. A una distancia alejada, se encuentran Ecuador, México, Argentina, Venezuela y Colombia (los cuales tienen valores muy similares de su Poder Naval total, ya que han basado su desarrollo naval en torno a patrullas oceánicas sin misiles embarcados), y en un último y muy alejado lugar se encuentra Cuba con una armada pequeña y de poco potencial.

Figura 6.

Poder Naval total

\begin{tabular}{lc}
\hline PAÍS & Poder Naval Total \\
\hline Chile & 20,37 \\
\hline Perú & 16,94 \\
\hline Brasil & 14,25 \\
\hline Ecuador & 10,7 \\
\hline México & 10,16 \\
\hline Argentina & 9,63 \\
\hline Venezuela & 9,19 \\
\hline Colombia & 8,53 \\
\hline Cuba & 2,87 \\
\hline
\end{tabular}

Fuente: elaboración propia. 
Con los valores anteriores, fue posible determinar en qué tipo de armada se encuentra clasificado cada país, las más altas: Chile, Perú y Brasil son del Tipo 6 (Till 2007, Vid Supra), lo que las ubica como Armadas de Defensa Territorial Marítima, las cuales tienen capacidad de negar el uso del mar al enemigo y presentar combate en su Zona Económica Exclusiva (ZEE). Ecuador, México, Argentina y Venezuela tienen Armadas de nivel 8, de la clase: Armadas de Vigilancia policial, con la capacidad de mantener el Estado de derecho en su ZEE contra la delincuencia organizada, pero no tienen la capacidad de negar el uso del mar a una fuerza Naval de otro Estado (Cuervo Vázquez 2014: 37-38). En la figura 8 se observa el Tipo de Armada de cada país analizado; conforme se va acercando al centro de la figura, la Armada va adquiriendo mayor Poder Naval total.

\begin{tabular}{lc}
\hline PAÍs & Tipo de Armada \\
\hline Brasil & 6 \\
\hline Chile & 6 \\
\hline Perú & 6 \\
\hline Argentina & 8 \\
\hline Colombia & 8 \\
\hline Ecuador & 8 \\
\hline México & 8 \\
\hline Venezuela & 8 \\
\hline Cuba & 9 \\
\hline
\end{tabular}

Fuente: elaboración propia.

El Modelo de medición del Poder Naval, permite también encontrar el valor del Índice de Riqueza Marítima Nacional: Brasil es el país que cuenta con más de $14 \%$ de la riqueza marítima de Latinoamérica, seguido por México con más del $12 \%$; de ahí, ningún otro país cuenta con más de $10 \%$ de la riqueza marítima de la región (Figura 9).

A partir del valor anterior (IRMN), se obtienen dos valores más, igualmente importantes: el índice de Defensa Marítima Policial, el cual nos indica a partir de la relación de su Poder Naval total y su índice de riqueza, qué porcentaje de esta riqueza está siendo protegida contra infractores de la ley y cuánto de este porcentaje se encuentra sin protección.

Cinco países cuentan con armadas capaces de proteger al 100\% a su riqueza marítima contra los infractores de la ley: Chile, Colombia, Ecuador, Perú y Venezuela. Argentina la protege en un $81,3 \%$, Brasil y Venezuela en un poco más del $70 \%$ y, por último, México, que con su armada actual alcanza a proteger un 62,9 $\%$ de sus riquezas marítimas. Las gráficas siguientes nos muestran los porcentajes de riqueza marítima sin protección actual contra infractores de la ley (conforme más se acerca el país al centro, mayor es el porcentaje sin protección, Figura 10).
Hacia una medición del poder naval en América Latina

Noé Cuervo Vázquez Marcos Pablo Moloeznik

Figura 8.

Tipos de Armada 


\begin{tabular}{lcc}
\hline PAÍs & \multicolumn{3}{c}{$\begin{array}{c}\text { Índice de Riqueza } \\
\text { Marítima }\end{array}$} \\
\hline Brasil & 18 & $14,17 \%$ \\
\hline México & 16 & $12,60 \%$ \\
\hline Argentina & 12 & $9,45 \%$ \\
\hline Chile & 9 & $7,09 \%$ \\
\hline Venezuela & 9 & $7,09 \%$ \\
\hline Colombia & 5 & $3,94 \%$ \\
\hline Perú & 5 & $3,94 \%$ \\
\hline Cuba & 4 & $3,15 \%$ \\
\hline Ecuador & 4 & $3,15 \%$ \\
\hline
\end{tabular}

Fuente: elaboración propia.

Figura 9.

Índice de Riqueza Marítima Nacional

Figura 10.

Índice de Defensa contra Infractores de la Ley

Figura 11.

Porcentaje de Riqueza Marítima sin proteger contra infractores de la ley
Índice de Defensa contra Infractores de la Ley

\begin{tabular}{lc}
\hline PAÍs & Índice de Defensa contra Infractores de la Ley \\
\hline Chile & 100 \\
\hline Colombia & 100 \\
\hline Ecuador & 100 \\
\hline Perú & 100 \\
\hline Venezuela & 100 \\
\hline Argentina & 81,3 \\
\hline Brasil & 78,6 \\
\hline Cuba & 74,6 \\
\hline México & 62,9 \\
\hline
\end{tabular}

Fuente: elaboración propia.

\begin{tabular}{lc}
\hline \multicolumn{1}{c}{ PAÍS } & \% Riqueza Marítima sin proteger contra infractores de la ley \\
\hline México & 37,1 \\
\hline Cuba & 25,4 \\
\hline Brasil & 21,4 \\
\hline Argentina & 18,7 \\
\hline Chile & 0 \\
\hline Colombia & 0 \\
\hline Ecuador & 0 \\
\hline Perú & 0 \\
\hline Venezuela & 0 \\
\hline
\end{tabular}

Fuente: elaboración propia. 
El último valor que se obtiene con el modelo, es el Índice de Defensa Naval, el cual nos permite relacionar las Capacidades Navales actuales con el índice de Riqueza Marítima Nacional, y obtener un porcentaje de defensa actual contra amenazas provenientes de otra fuerza naval. Su valor nos proporciona también el porcentaje de riqueza marítima sin proteger.

Los valores obtenidos permiten observar que Chile, Ecuador y Perú cuentan con armadas capaces de proteger ${ }^{13}$ al $100 \%$ a sus riquezas marítimas contra otro Estado agresor. Venezuela tiene un índice de protección del 73 \%, Brasil, Colombia, Cuba y Argentina cuentan con un porcentaje de protección alrededor del $50 \%$ y sólo México cuenta con un valor muy bajo de menos de $10 \%$ de protección de sus riquezas contra otra fuerza naval de un Estado agresor (Figura 12).

\begin{tabular}{lc}
\hline \multicolumn{1}{c}{ PAÍS } & Índice de Defensa contra Flota Naval \\
\hline Chile & 100 \\
\hline Ecuador & 100 \\
\hline Perú & 100 \\
\hline Venezuela & 73 \\
\hline Brasil & 54,5 \\
\hline Colombia & 49,8 \\
\hline Cuba & 42,3 \\
\hline Argentina & 41,8 \\
\hline México & 8,6 \\
\hline
\end{tabular}

Fuente: elaboración propia.

\begin{tabular}{lc}
\hline \multicolumn{1}{c}{ PAÍS } & \%Riqueza Marítima sin proteger contra Flota Naval \\
\hline México & 91,4 \\
\hline Argentina & 58,2 \\
\hline Cuba & 57,7 \\
\hline Colombia & 50,2 \\
\hline Brasil & 45,5 \\
\hline Chile & 0 \\
\hline Ecuador & 0 \\
\hline Perú & 0 \\
\hline Venezuela & 0 \\
\hline
\end{tabular}

Fuente: elaboración propia.

${ }^{13}$ Esto se refiere solo a la proporción entre sus capacidades navales actuales y su riqueza marítima, en función de datos cuantitativos. Queda claro que la defensa de un territorio contra otro Estado enemigo pasa también por datos cualitativos como voluntad de lucha y resistencia, experiencia en combate, nivel de capacitación y adiestramiento, unidad nacional, fortaleza ideológica, entre otros intangibles, variables que no son abordadas en el presente estudio.
Hacia una medición del poder naval en América Latina

Noé Cuervo Vázquez Marcos Pablo Moloeznik

\section{Figura 12.}

Índice de Defensa contra otra Flota Naval

\section{Figura 13.}

Porcentaje de Riqueza Marítima sin proteger contra otra Flota Naval 
A partir de la aplicación del modelo y la descripción previa de las armadas, se puede observar que en Latinoamérica conviven tres paradigmas del poder naval: el brasileño, el chileno y el colombiano (Moloeznik 2013). Sólo Brasil y Chile (quien resultó el mejor calificado según el modelo), aspiran a dar un salto cualitativo y proyectar su poder nacional en apoyo a sus intereses marítimos y a su política exterior, es decir, al activo papel que persiguen en el concierto de las naciones.

De conformidad con The Military Balance (2013), la armada brasileña (a pesar de salir en tercera posición), tiene un programa muy ambicioso de adquisición de fragatas, submarinos y patrulleros de altura. Se encuentran en construcción, entre otros, submarino nuclear ordenado en 2009 para estar listo en 2025 así como cuatro submarinos de ataque de propulsión diésel que pretenden estar listos en el 2017.

Chile es un país esencialmente marítimo (su visión operacional actual de su armada lo colocó en el primer sitio); el mar es vital para su desarrollo y fundamental para la subsistencia de su población, manifiesta la voluntad y compromiso internacional de ejercer presencia en el espacio de la alta mar aledaño a su zona económica exclusiva, denominado "mar presencial", donde ha asumido una serie de responsabilidades internacionales relacionadas con la protección de la vida humana en el mar, el control del tráfico marítimo y la conservación del medio ambiente (Moloeznik 2016).

La Armada Nacional de Colombia (que representa al tercer paradigma) se encuentra inadecuadamente equipada para hacer frente a las responsabilidades como fuerza bioceánica (mar Caribe y Océano Pacífico). Recientemente, sus esfuerzos de modernización de la flota se concentraron en los buques patrulleros de río y aviación de patrullaje, así como en su infantería de marina, instrumento ad hoc para combatir la insurgencia y el narcotráfico.

El modelo colombiano (en donde se debe incluir a México) constituye la antítesis del brasileño, según la propuesta de paradigmas de Moloeznik (2016). A pesar de su condición de país bioceánico, ha otorgado prioridad a la amenaza del llamado narcoterrorismo y, al igual que México, ambos otorgan preeminencia a las lanchas patrulleras costeras, interceptoras y oceánicas, en detrimento de destructores, cruceros, fragatas e incluso corbetas $y$, asimismo, presentan una infantería de marina sobredimensionada.

\section{Conclusiones y soluciones para el caso mexicano}

Las conclusiones que a continuación se exponen se desprenden de los resultados obtenidos y permiten establecer lo siguiente:

- Las Capacidades Navales actuales de México se encuentran en el último sitio de los nueve países seleccionados como muestra. En contraste, sus Capacidades Navales potenciales son las más altas de la región, lo que explica que el Poder Naval total de México se ubique en la posición número cinco de los nueve evaluados respecto a su Poner Naval total. 
- La clasificación de los países con el mayor Índice de Poder Naval en Latinoamérica es: primer lugar Chile, seguido por Perú, Brasil, Ecuador, México, Argentina, Venezuela, Colombia y Cuba, consecutivamente.

- El país con el mayor índice de Capacidades Navales potenciales es México, seguido de Brasil.

- El país con el mayor índice de Capacidades Navales actuales es Chile, seguido de Perú.

- El país con el mayor índice de Riqueza Marítima Nacional es Brasil, seguido de México.

- El país con el mayor porcentaje de Riqueza Marítima Nacional sin proteger, es México, esto en función de la relación entre su Poder Naval y la cantidad de Riqueza Marítima del País.

- Los buques más importantes, de acuerdo a su incidencia en el valor total del modelo empleado, fueron los buques tipo fragata, corbeta y patrulla SAAR 4.5 (Aliya), por encima de buques de tipo mayor.

- Las Armadas de Chile, Perú y Brasil son del tipo seis (Armadas de Defensa territorial Marítima), las Armadas de Ecuador, México, Argentina, Venezuela y Colombia son Armadas del tipo ocho (Armadas de Vigilancia policial) y la Armada de Cuba es del tipo nueve (Armadas simbólicas). El resto de las Armadas de Latinoamérica no tienen recursos para ser analizadas dentro del modelo propuesto.

- Los misiles más empleados en la región son los tipo superficie-superficie Exocet, seguidos por los Harpoon; en tanto en los del tipo superficie-aire fueron los Aspide seguidos por los Sea Wolf.

- En relación a México, se puede concluir que la enorme Riqueza Marítima Nacional que tiene en su territorio, solo por debajo de Brasil, obliga a tener un Poder Naval total mayor, a partir de aprovechar el importante potencial con que cuenta.

- La posición de México se puede resumir de la siguiente manera: es el país con el mayor índice de Capacidades Navales potenciales, con el índice más bajo de Capacidades Navales actuales, se encuentra en el quinto sitio en cuanto a su Poder Naval total, es el segundo lugar en cuanto al índice de Riqueza Marítima Nacional, pero tiene, de acuerdo al modelo utilizado, casi un $40 \%$ de su riqueza marítima desprotegida contra infractores de la ley y más del $90 \%$ de su riqueza marítima desprotegida contra otra fuerza naval en caso de un ataque en estos momentos.

- Como conclusión final se identificó que en la actualidad, para aumentar el Poder Naval de un Estado, no sólo son necesarios los tipos de buques, ni portaaviones, ni submarinos; la clave para una armada pequeña o mediana son los misiles y sus sistemas de armas. Las unidades de superficie constituyen la columna vertebral de una armada, el contar con misiles lo convierte en un multiplicador de fuerza.

A partir del análisis realizado y las conclusiones obtenidas, se pudo observar que los buques que están siendo más preponderantes en el Poder Naval
Hacia una medición del poder naval en América Latina

Noé Cuervo Vázquez Marcos Pablo Moloeznik 
total en Latinoamérica son los de mediano desplazamiento, principalmente del tipo fragatas y corbetas, pero armadas con misiles tanto del tipo superficiesuperficie y del tipo superficie-aire.

La propuesta de solución que se plantea en el presente trabajo de investigación es la continuación de la construcción de los buques patrulla oceánica polivalentes y multipropósito, con la variante de integrar en su armamento sistemas de armas y lanzadores de misiles tanto de superficie-superficie y de superficie-aire, junto a una dotación importante de misiles en cada unidad de superficie; asimismo, iniciar en el Instituto de Investigación y Desarrollo de la Armada de México un programa de construcción de prototipos de misiles como armamento fundamental en las armadas modernas.

Bajo estas dos premisas, basadas primordialmente en el empleo de misiles a bordo de las nuevas patrullas oceánicas, México podrá aumentar significativamente el valor de las Capacidades Navales actuales y por ende, el valor del Poder Naval total de la Armada Nacional.

\section{Bibliografía}

Athieu H. (2012), Anuario 2012 de la Seguridad Regional en América Latina y el Caribe, FES Seguridad, Bogotá.

Centro de Estudios Superiores Navales (CESNAV) (2003), Poder Marítimo Mexicano, CESNAV, México.

Chant C. (2006), Barcos de Guerra, LIBSA, Madrid.

Colom G. (2009), Entre la revolución y la transformación en los asuntos militares y la configuración de los pilares estratégicos de Estados Unidos para el siglo XXI, tesis doctoral, Instituto Universitario Gutierrez Mellado, Madrid.

Couteau-Begarie H. (2010), La potencia marítima, Ediciones Ejército, Madrid.

Crisher B., Souva M. (15 de septiembre de 2013), Power at sea: a naval power dataset, 1865 2011. Universidad de Florida, Florida, Estados Unidos de América.

Cuervo Vázquez N. (2014), El Poder Naval en Latinoamérica: Análisis correlacional del Poder Naval de México, Centroamérica y Sudamérica. (Construcción de un instrumento para medir el Poder Naval de un Estado), tesis de maestría, Secretaría de Marina, Armada de México, Centro de Estudios Superiores Navales, México, D.F.

de Saint Salvy A. F. (1994), Concevoir la marine: un art difficile, "Défense Nationale", Revue mensuelle, Mai 1994, Dossier La marine nationale, Comité d’Études de Défense National, Paris.

Diez O. (2006), Buques, Submarinos y Portaaviones, UDYAT, Madrid.

Friedman N. (2006), The Naval Institute Guide to Word Naval Weapons, Naval Institute Press, Annapolis.

Fuller M. (2013), IHS Jane's Weapons Naval, Polestar Wheatons, Reino Unido.

Gray S. (2006), La pujanza del Poder Naval, Ministerio de Defensa, Madrid. 
Gresham J. D. (2005), SEAPOWER, El dominio del mar, Océano, México.

Hardy D. (2005), La proyección del poder militar a través del mar; máxima contribución naval del accionar conjunto, "REVISMAR", no 6, 2005, pp. 517-528.

Hill J. (1990), Estrategia marítima para potencias medianas, Instituto de Publicaciones Navales, Buenos Aires.

International Institute for Strategic Studies (IISS) (2013), The Military Balance 2013, Routledge, London.

Jackson R. (2011), Barcos de Guerra, Edimat Libros S.A, Madrid.

Martinez D. (2009), Poderío marítimo, Escuela de Guerra Naval, Buenos Aires.

Mey C. (14 de octubre de 2013), El equilibrio naval durante la época del apostadero. Historia y arqueología naval, disponible en: Histamar, http://www.histamar.com.ar

Moloeznik M. (2009), Hacia un marco teórico y analítico del poder naval en México. Contribución doctrinaria al desarrollo de la Armada de México, Análisis, Guadalajara.

Moloeznik M. (2013), La Armada de México frente a sus pares de América Latina, "Revista del CESLA", no 14, 2011, pp. 39-71.

Moloeznik M. (2016), Derroteros y paradigmas navales en Latinoamérica, en: Przeobrażenia geopolityczne i nowe zagrożenia w Ameryce Łacińskiej - Transformaciones geopolíticas y nuevas amenazas en América Latina, K. Krzywicka, P. Trefler (eds. y coords.), Estudios Iberoamericanos de la UMCS, vol. IV, Editorial de la Universidad Maria Curie-Skłodowska, Lublin.

Ness L. (2013), Jane's Weapons Ammunition, Polestar Wheatons, Reino Unido.

Payne C. M. (2010), Principles of Naval Weapons Systems, Naval Institute Press, Annapolis.

RESDAL (2012), Atlas comparativo de la Defensa en América Latina y el Caribe, RESDAL, Buenos Aires.

Ricardi C. (2008), Tesis: Cuantificar intereses marítimos, Escuela de Guerra Naval, Montevideo.

Saunders S. (14 de octubre de 2013), Analysis 2013 Global naval developments trends and outlook, obtenido de IHS Jane's: http://www.ihs.com.jfs

Saunders S. (2013), IHS Jane's Fighting Ships 2013-2014, JANE'S HIS, Virginia.

SIPRI (2010), SIPRI Yearbook 2010, SIPRI - UNAM, Estocolmo.

Sheina R. L. (1991), Iberoamérica. Una Historia Naval 1810-1987, Editorial San Martín, Madrid.

Till G. (2007), Poder Marítimo: una guía para el siglo XXI, Instituto de Publicaciones Navales, Buenos Aires.

U.S. Military, Department of Defense, U.S. Navy, World Spaceflight News (2012), Ultimate illustrated navy equipment.

Vego M. (2009), Estrategia Naval y operaciones en aguas restringidas, Ministerio de Defensa, Madrid.

Vilanova C. B. (2006), El hombre y la mar, Grupo Cultural, Madrid.

Vilanova C. B. (2012), LHD La máxima proyección estratégica, Real del Catorce Editores, Madrid.

Wragg D. (2012), Word Sea Power Guide, Pen \& Sword, Maritime, South Yorkshire.
Hacia una medición del poder naval en América Latina

Noé Cuervo Vázquez Marcos Pablo Moloeznik 